\title{
Triple point numbers of surface-links and symmetric quandle cocycle invariants
}

\author{
KANAKO OSHIRO
}

\begin{abstract}
For any positive integer $n$, we give a 2-component surface-link $F=F_{1} \cup F_{2}$ such that $F_{1}$ is orientable, $F_{2}$ is non-orientable and the triple point number of $F$ is equal to $2 n$. To give lower bounds of the triple point numbers, we use symmetric quandle cocycle invariants.
\end{abstract}

57Q45; 18G99, 55N99, 57Q35

\section{Introduction}

A surface-link is a closed surface smoothly embedded in $\mathbb{R}^{4}$. Two surface-links $F$ and $F^{\prime}$ are assumed to be the same if and only if there exists an ambient isotopy $\left\{h_{t}\right\}$ of $\mathbb{R}^{4}$ such that $h_{1}(F)=F^{\prime}$. When $F$ and $F^{\prime}$ are oriented, it is assumed that $\left.h_{1}\right|_{F}: F \rightarrow F^{\prime}$ is an orientation-preserving homeomorphism. In particular, when a surface-link is connected, we call it a surface-knot.

The triple point number of a surface-link $F$ is defined by the smallest number of the triple points among all the diagrams of $F$, and we denote it by $t(F)$. There are several studies on triple point numbers. For example, quandle cocycle invariants (see Carter, Jelsovsky, Kamada, Langford and Saito [1]) are used to give lower bounds of triple point numbers of orientable surface-links; for example, Satoh and Shima [14] determined the triple point number of the 2-twist-spun trefoil to be four, and Hatakenaka [4] gave a lower bound for the triple point number of the 2-twist-spun figure-eight knot. By a geometric argument about normal Euler numbers, Satoh [12] gave the following theorem:

Theorem 1.1 (Satoh [12]) For any positive integer $n$, there exists a 2-component surface-link $F=F_{1} \cup F_{2}$ such that (i) each $F_{i}$ is a non-orientable surface-knot with the Euler characteristic $\chi\left(F_{i}\right)=2-n$, and (ii) $t(F)=2 n$.

In Section 4, we show a method which gives lower bounds for the triple point numbers of surface-links by using the symmetric quandle cocycle invariants (see Kamada [7] 
and Kamada-Oshiro [8]). We remark that by the symmetric quandle cocycle invariants, we can give alternative proof of Theorem 1.1. Using new examples of surface-links, we can also prove the following theorem which is analogous to Theorem 1.1:

Theorem 1.2 For any positive integer $n$, there exists a 2-component surface-link $F=F_{1} \cup F_{2}$ such that

(i) $F_{1}$ is an orientable surface-knot with $\chi\left(F_{1}\right)=0$,

(ii) $\quad F_{2}$ is a non-orientable surface-knot with $\chi\left(F_{2}\right)=2-2 n$, and

(iii) $t(F)=2 n$.

By a connected sum of the surface-link which Satoh used for Theorem 1.1 and an orientable surface-knot, the following was given in [8]: For any positive integers $m$ and $n$ with $m \equiv n(\bmod 2)$ and $m \geq n$, there is a surface-link $F=F_{1} \cup F_{2}$ such that $F_{1}$ is a non-orientable surface with $\chi\left(F_{1}\right)=2-m, F_{2}$ is a non-orientable surface with $\chi\left(F_{2}\right)=2-n$ and $t(F)=2 n$. For surface-links composed of two non-orientable surfaces, we give the following theorem:

Theorem 1.3 For any positive integer $n$ and for any integer $m$ with $m \geq 3$, there is a surface-link $F=F_{1} \cup F_{2}$ such that

(i) $F_{1}$ is a non-orientable surface with $\chi\left(F_{1}\right)=2-m$,

(ii) $\quad F_{2}$ is a non-orientable surface with $\chi\left(F_{2}\right)=2-2 n$, and

(iii) $t(F)=2 n$.

The paper is organized as follows. In Sections 2 and 3, we recall symmetric quandles, symmetric quandle 3-cocycles, and surface-link invariants with symmetric quandles introduced in $[7 ; 8]$. In Section 4 , we show a method to estimate the triple point numbers of surface-links by using the symmetric quandle invariants. Theorems 1.2 and 1.3 are proved by giving new examples of surface-links in Section 5. In Section 6, we show several results which can be obtained by using our method for estimating triple point numbers.

\section{Symmetric quandles and their cocycles}

A quandle (see Fenn and Rourke [3], Joyce [5] or Matveev [10]) is a set $X$ with a binary operation $(x, y) \mapsto x^{y}$ such that

(i) for any $x \in X$, it holds that $x^{x}=x$, 
(ii) for any $x, y \in X$, there exists a unique $z \in X$ such that $z^{y}=x$, and

(iii) for any $x, y, z \in X$, it holds that $\left(x^{y}\right)^{z}=\left(x^{z}\right)^{\left(y^{z}\right)}$.

We denote by $x^{y^{-1}}$ the element $z$ given in the condition (ii). For a quandle $X$, a good involution $\rho$ of $X[7 ; 8]$ means an involution of $X$ such that

(i) for any $x, y \in X, \rho\left(x^{y}\right)=\rho(x)^{y}$, and

(ii) for any $x, y \in X, x^{\rho(y)}=x^{y^{-1}}$.

A pair of a quandle and a good involution is called a symmetric quandle.

Let $(X, \rho)$ be a symmetric quandle, and $A$ an abelian group. A homomorphism $\theta: \mathbb{Z}\left(X^{3}\right) \rightarrow A$ is a symmetric quandle 3 -cocycle of $(X, \rho)$ if the following conditions are satisfied:

(i) For any $(a, b, c, d) \in X^{4}$,

$\theta(a, c, d)-\theta\left(a^{b}, c, d\right)-\theta(a, b, d)+\theta\left(a^{c}, b^{c}, d\right)+\theta(a, b, c)-\theta\left(a^{d}, b^{d}, c^{d}\right)=0$,

(ii) for any $(a, b) \in X^{2}, \theta(a, a, b)=0$ and $\theta(a, b, b)=0$, and

(iii) for any $(a, b, c) \in X^{3}$,

$$
\theta(a, b, c)+\theta(\rho(a), b, c)=0, \quad \theta(a, b, c)+\theta\left(a^{b}, \rho(b), c\right)=0
$$

and $\theta(a, b, c)+\theta\left(a^{c}, b^{c}, \rho(c)\right)=0$.

Here, $\mathbb{Z}\left(X^{3}\right)$ is the free $\mathbb{Z}$-module generated by all the elements of $X^{3}=X \times X \times X$. Notice that a symmetric quandle 3 -cocycle of $(X, \rho)$ is a 3 -cocycle of the cochain complex defined for the symmetric quandle $(X, \rho)$ in $[7 ; 8]$.

For any element $k$ in $\mathbb{Z}$, we use the same symbol $k$ to indicate the element $[k]$ in $\mathbb{Z}_{2}$, and any element of $\mathbb{Z}_{2} \oplus \mathbb{Z}$ is denoted by a form $\alpha \oplus \beta$, where $\alpha$ is the entry of $\mathbb{Z}_{2}$, and $\beta$ is the entry of $\mathbb{Z}$.

Example 2.1 The set $\{0,1, \cdots, n-1\}$ with the operation $x^{y} \equiv 2 y-x(\bmod n)$ for any $x, y \in\{0,1, \cdots, n-1\}$ is a quandle, which is called a dihedral quandle of order $n$. All of the good involutions of a dihedral quandle are determined in [8]. Let $X$ be the dihedral quandle $\{0,1,2,3\}$ of order 4 . The involution $\rho: X \rightarrow X$ defined by $\rho(0)=2$ and $\rho(1)=3$, is a good involution of $X$. Define a map $\theta: X^{3} \rightarrow \mathbb{Z}_{2} \oplus \mathbb{Z}$ such that

$$
\theta(a, b, c)=\left\{\begin{aligned}
& 0 \oplus 1 \quad(a, b, c)=(0,1,0),(0,3,0),(2,1,2),(2,3,2), \\
&(1,0,3),(1,2,3),(3,0,1),(3,2,1), \\
& 0 \oplus(-1)(a, b, c)=(0,1,2),(0,3,2),(2,1,0),(2,3,0), \\
&(1,0,1),(1,2,1),(3,0,3),(3,2,3), \\
& 0 \oplus 0 \quad \text { otherwise. }
\end{aligned}\right.
$$


Then the the linear extension $\theta: \mathbb{Z}\left(X^{3}\right) \rightarrow \mathbb{Z}_{2} \oplus \mathbb{Z}$ is a symmetric quandle 3-cocycle of $(X, \rho)$.

Example 2.2 Let $X=\{0,1,2\}$ be the quandle such that

$$
\begin{aligned}
& 0^{0}=0, \quad 0^{1}=0,0^{2}=0, \\
& 1^{0}=2,1^{1}=1,1^{2}=1, \\
& 2^{0}=1,2^{1}=2,2^{2}=2 .
\end{aligned}
$$

The involution $\rho: X \rightarrow X$ defined by $\rho(0)=0$ and $\rho(1)=2$, is a good involution of $X$. Define a map $\theta: X^{3} \rightarrow \mathbb{Z}_{2} \oplus \mathbb{Z}$ such that

$$
\theta(a, b, c)= \begin{cases}1 \oplus 0 & (a, b, c)=(0,1,0),(0,2,0) \\ 0 \oplus 1 & (a, b, c)=(1,0,2),(2,0,1) \\ 0 \oplus(-1) & (a, b, c)=(1,0,1),(2,0,2) \\ 0 \oplus 0 & \text { otherwise. }\end{cases}
$$

Then the linear extension $\theta: \mathbb{Z}\left(X^{3}\right) \rightarrow \mathbb{Z}_{2} \oplus \mathbb{Z}$ is a symmetric quandle 3-cocycle of $(X, \rho)$.

\section{Symmetric quandle cocycle invariants}

Let $D$ be a diagram in $\mathbb{R}^{3}$ of a surface-link $F$ in $\mathbb{R}^{4}$, where the lower sheets are divided along double point curves to indicate crossing information. We divide over-sheets along the double point curves and we call the sheets of the result semi-sheets of $D$. Note that every semi-sheet is orientable even if $F$ is non-orientable, see Kamada [6].

For a symmetric quandle $(X, \rho)$, we say that an assignment of a normal orientation and an element of $X$ to each semi-sheet of $D$ satisfies the coloring conditions if it satisfies the following:

(i) Suppose that two adjacent semi-sheets coming from an over-sheet of $D$ about a double point curve are labeled by $x_{1}$ and $x_{2}$. If the normal orientations are coherent then $x_{1}=x_{2}$, otherwise $x_{1}=\rho\left(x_{2}\right)$. See the top row of Figure 1 .

(ii) Suppose that two adjacent semi-sheets $S_{1}$ and $S_{2}$ coming from under-sheets about a double point curve are labeled by $x_{1}$ and $x_{2}$, and that one of the two semi-sheets coming from an over-sheet of $D$, say $S_{3}$, is labeled by $x_{3}$. We assume that the normal orientation of $S_{3}$ points from $S_{1}$ to $S_{2}$. If the normal orientations of $S_{1}$ and $S_{2}$ are coherent, then $x_{1}^{x_{3}}=x_{2}$, otherwise $x_{1}^{x_{3}}=\rho\left(x_{2}\right)$. See the bottom row of Figure 1. 

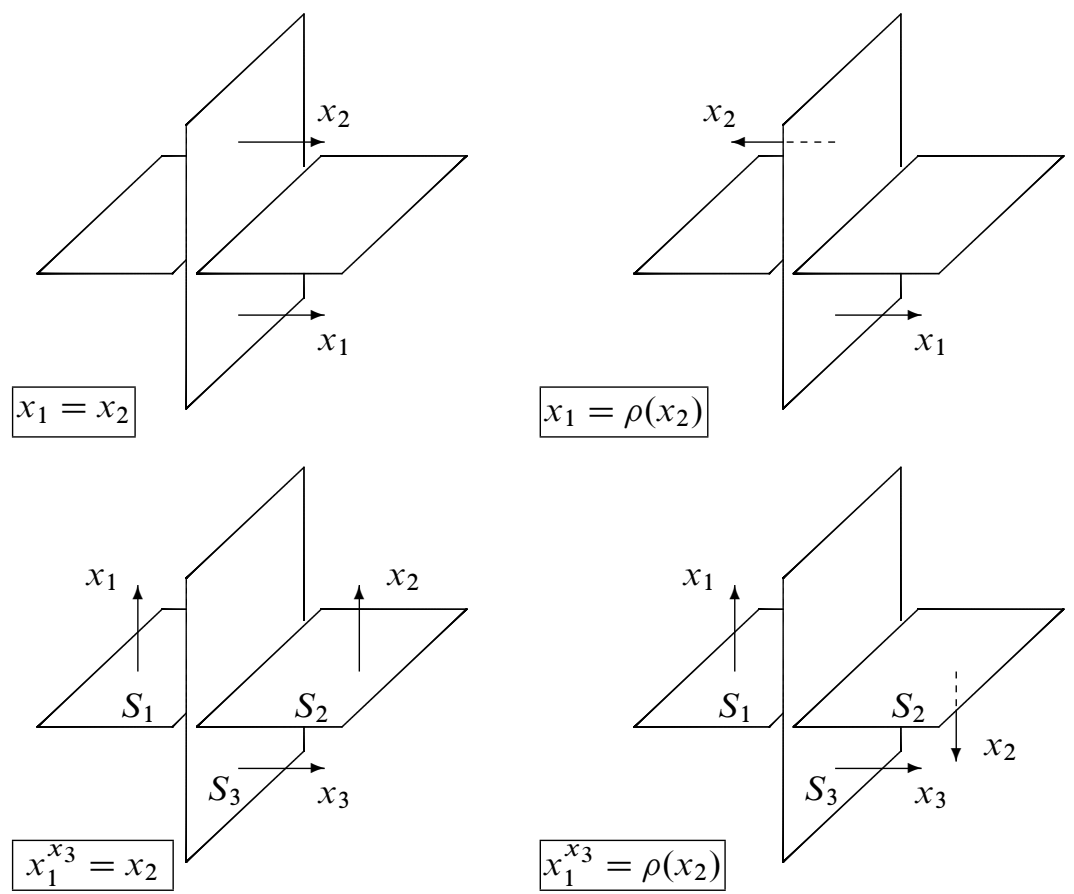

Figure 1

An $(X, \rho)$-coloring of $D$ is the equivalence class of an assignment of normal orientations and elements of $X$ to the semi-sheets of $D$ satisfying the coloring conditions. Here, the equivalence relation is generated by basic inversions, that is, a basic inversion reverses the normal orientation of a semi-sheet and changes the element $x$ assigned the sheet by $\rho(x)$. See Figure 2 .
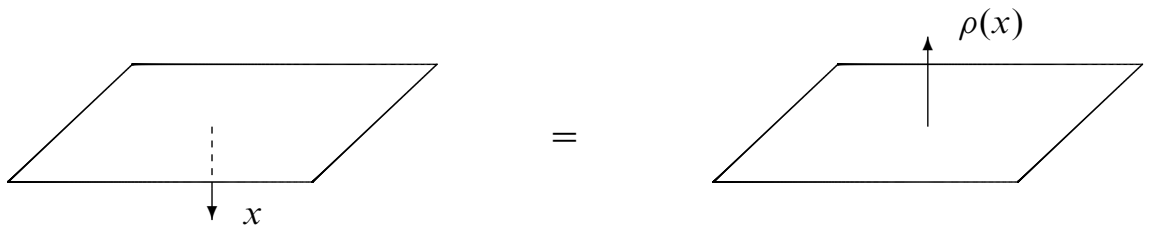

Figure 2

We call a diagram with an $(X, \rho)$-coloring $C_{D}$ an $(X, \rho)$-colored diagram and denote it by $\left(D, C_{D}\right)$. 
Let $\left(D, C_{D}\right)$ and $\left(D^{\prime}, C_{D^{\prime}}\right)$ be $(X, \rho)$-colored diagrams of a surface-link $F$. We say that $\left(D, C_{D}\right)$ and $\left(D^{\prime}, C_{D^{\prime}}\right)$ (or the $(X, \rho)$-colorings $C_{D}$ of $D$ and $C_{D^{\prime}}$ of $D^{\prime}$ ) are equivalent if they are related by a finite sequence of Roseman moves (see Roseman [11], and also Carter and Saito [2]) over which the colorings extend. We call the equivalence class of $\left(D, C_{D}\right)$ an $(X, \rho)$-coloring of $F$. An $(X, \rho)$-colored surface-link $(F, C)$ is a surface-link $F$ equipped with an $(X, \rho)$-coloring $C$.

Let $\left(D, C_{D}\right)$ be an $(X, \rho)$-colored diagram of an $(X, \rho)$-colored surface-link $(F, C)$. Let $\theta: \mathbb{Z}\left(X^{3}\right) \rightarrow A$ be a symmetric quandle 3-cocycle of $(X, \rho)$. For a triple point of $D$, define the $\theta$-weight as follows: Choose one of eight 3-dimensional complementary regions around the triple point and call the region a specified region. There exist 12 semi-sheets around the triple points. Let $S_{T}, S_{M}$ and $S_{B}$ be the three of them that face the specified region, where $S_{T}, S_{M}$ and $S_{B}$ are in the top sheet, the middle sheet and the bottom sheet at the triple point, respectively. Let $n_{T}, n_{M}$ and $n_{B}$ be the normal orientations of $S_{T}, S_{M}$ and $S_{B}$ which point away from the specified region. Let $x, y$ and $z$ be the elements of $X$ assigned to the semi-sheets $S_{T}, S_{M}$ and $S_{B}$ with the normal orientations $n_{T}, n_{M}$ and $n_{B}$, respectively. The $\theta$-weight of the triple point is defined by $\varepsilon \theta(z, y, x)$, where $\varepsilon$ is +1 (or -1 ) if the triple of the normal orientations $\left(n_{T}, n_{M}, n_{B}\right)$ does (or does not) match with the orientation of $\mathbb{R}^{3}$. The sign of the triple point as shown in Figure 3 is positive.

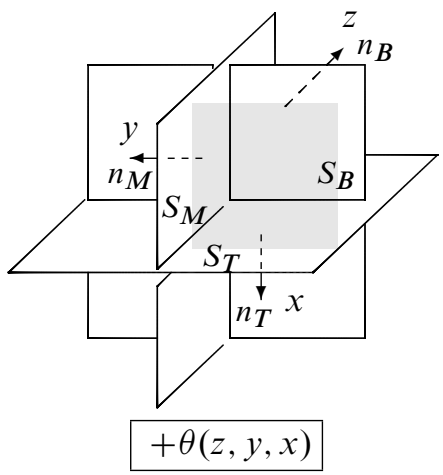

Figure 3

Define $\theta\left(D, C_{D}\right)$ by

$$
\theta\left(D, C_{D}\right)=\sum_{\tau}(\theta \text {-weight of } \tau) \in A,
$$

where $\tau$ runs over all the triple points of $D$. 
Theorem 3.1 (Kamada and Oshiro [8]) The value $\theta\left(D, C_{D}\right)$ is an invariant of an $(X, \rho)$-colored surface-link $(F, C)$.

We denote $\theta\left(D, C_{D}\right)$ by $\theta(F, C)$.

\section{Estimates of triple point numbers}

For non-negative integers $s$ and $t$, let $A_{s, t}$ denote the direct sum of $s$ copies of $\mathbb{Z}_{2}$ and $t$ copies of $\mathbb{Z}$, that is, $A_{s, t}=\left(\mathbb{Z}_{2}\right)^{s} \oplus(\mathbb{Z})^{t}$. Every element of $A_{s, t}$ has a form $\left(\alpha_{1} \oplus \cdots \oplus \alpha_{s}\right) \oplus\left(\beta_{1} \oplus \cdots \oplus \beta_{t}\right)$, where $\alpha_{i}$ is the entry of $i$ th $\mathbb{Z}_{2} \quad(1 \leq i \leq s)$, and $\beta_{j}$ is the entry of $j$ th $\mathbb{Z}(1 \leq j \leq t)$. We denote by $p_{i}$ and $q_{j}$ the elements of $A_{s, t}$ whose entries are all zeros except $\alpha_{i}=1$ and $\beta_{j}=1$, respectively.

Let $(X, \rho)$ be a symmetric quandle, and $\theta: \mathbb{Z}\left(X^{3}\right) \rightarrow A_{s, t}$ a 3 -cocycle of $(X, \rho)$. We consider the following condition for $\theta$ :

(*) For any generator $(a, b, c) \in X^{3}$ of $\mathbb{Z}\left(X^{3}\right)$, it holds that

$$
\theta(a, b, c) \in\left\{0, p_{i}, \pm q_{j} \mid 1 \leq i \leq s, 1 \leq j \leq t\right\} .
$$

We remark that the symmetric quandle 3-cocycles given in Examples 2.1 and 2.2 satisfy the condition $(*)$.

Theorem 4.1 Let $\theta$ be a 3-cocycle of a symmetric quandle $(X, \rho)$ with the condition (*). If the invariant $\theta(F, C)$ of a surface-link $F$ with an $(X, \rho)$-coloring $C$ is equal to $\left(\alpha_{1} \oplus \cdots \oplus \alpha_{s}\right) \oplus\left(\beta_{1} \oplus \cdots \oplus \beta_{t}\right)$, then we have $t(F) \geq \sum_{i=1}^{s} \alpha_{i}+\sum_{j=1}^{t}\left|\beta_{j}\right|$, where the sum is taken in $\mathbb{Z}$ by regarding $\alpha_{k}=0$ or 1 as an element of $\mathbb{Z}$.

Proof We take any $(X, \rho)$-colored diagram $\left(D, C_{D}\right)$ of $(F, C)$. Let $t(D)$ denote the number of triple points of $D$, and $m_{i}(1 \leq i \leq s), n_{j}$ and $n_{j}^{\prime}(1 \leq j \leq t)$ the number(s) of triple points whose $\theta$-weights are $p_{i}, q_{j}$ and $-q_{j}$, respectively.

Since the $\theta$-weight of any triple point of $D$ is one of $0, p_{i}, q_{j}$, and $-q_{j}$, it holds that

$$
\begin{aligned}
\theta\left(D, C_{D}\right) & =\sum_{i=1}^{s} m_{i} p_{i}+\sum_{j=1}^{t} n_{j} q_{j}+\sum_{j=1}^{t} n_{j}^{\prime}\left(-q_{j}\right) \\
& =\left(m_{1} \oplus \cdots \oplus m_{s}\right) \oplus\left(\left(n_{1}-n_{1}^{\prime}\right) \oplus \cdots \oplus\left(n_{t}-n_{t}^{\prime}\right)\right) .
\end{aligned}
$$

Hence, we have $\alpha_{i} \equiv m_{i}(\bmod 2)$ and $\beta_{j}=n_{j}-n_{j}^{\prime}$ by assumption. Since $\alpha_{i} \leq m_{i}$ and $\left|\beta_{j}\right| \leq n_{j}+n_{j}^{\prime}$, it holds that

$$
\sum_{i=1}^{s} \alpha_{i}+\sum_{j=1}^{t}\left|\beta_{j}\right| \leq \sum_{i=1}^{s} m_{i}+\sum_{j=1}^{t}\left(n_{j}+n_{j}^{\prime}\right) \leq t(D) .
$$




\section{Proofs of Theorems 1.2 and 1.3}

In this section, we give surface-links which satisfy Theorems 1.2 and 1.3.

Let $F$ be a surface-link in $\mathbb{R}^{4}=\left\{(x, y, z, t) \in \mathbb{R}^{4}\right\}$ whose motion picture is given in Figure 4. It is composed of an unknotted torus $F_{1}$ and an unknotted, non-orientable surface $F_{2}$ with $\chi\left(F_{2}\right)=2-2 n$. Notice that in Figure 4, the deformations from (i) to (ii) and from (iii) to (iv) are the isotopic deformations corresponding to $n$ Reidemeister moves of type III, respectively. The other isotopies are obtained by Reidemeister moves I and II only.

Let $D$ be the diagram obtained by the projection $\pi: \mathbb{R}^{4} \rightarrow \mathbb{R}^{3}$ with $\pi(x, y, z, t) \mapsto$ $(x, y, 0, t)$. Instead of illustrating the whole of $D$, we use the one-parameter family $\left\{D \cap \mathbb{R}^{2}[t]\right\}_{t \in \mathbb{R}}$, where $\mathbb{R}^{2}[t]=\{(x, y, 0, t) \mid x \in \mathbb{R}, y \in \mathbb{R}\}$.

Proof of Theorem 1.2 We will prove that the surface-link $F$ constructed as above satisfies $t(F)=2 n$. It is not difficult to see that $\chi\left(F_{2}\right)=2-2 n$.

Let $(X, \rho)$ and $\theta$ be the symmetric quandle and the symmetric quandle 3-cocycle given in Example 2.2. We define an $(X, \rho)$-coloring $C$ for $D$ such that (i) any semi-sheet of $F_{1}$ is assigned by $0 \in X$ with any normal orientation, and (ii) the semi-sheet of $F_{2}$ marked by $*$ in $\mathbb{R}^{2}[-2]$ is assigned by $1 \in X$ with the orientation as in the figure, which can be extended to any other semi-sheets of $F_{2}$ uniquely.

Between the stills (i) and (ii) in Figure 4, the Reidemeister moves of type III arise $n$ times and each move is depicted in Figure 5. Each Reidemeister move of type III corresponds to a triple point whose $\theta$-weight is $-\theta(2,0,2)=0 \oplus 1$. Between the stills (iii) and (iv) in Figure 4, the Reidemeister moves of type III arise $n$ times and each move is depicted in Figure 6. Each Reidemeister move of type III corresponds to a triple point whose $\theta$-weight is $\theta(1,0,2)=0 \oplus 1$. Therefore, $\theta\left(E^{(n)}, C\right)$ is equal to $0 \oplus 2 n$. By Theorem 4.1, $t\left(E^{(n)}\right) \geq 2 n$.

Proof of Theorem 1.3 Let $F=F_{1} \cup F_{2}$ be the surface-link as above, and $K$ an unknotted non-orientable surface-knot with $\chi(K)=4-m(m \geq 3)$. We denote by $F \sharp K=\left(F_{1} \sharp K\right) \cup F_{2}$ the connected sum of $F_{1} \subset F$ and $K$. It follows by definition that $\chi\left(F_{1} \sharp K\right)=2-m$ and $t(F \sharp K) \leq 2 n$.

On the other hand, the $(X, \rho)$-coloring $C$ for $F$ in the proof of Theorem 1.2 is extended to that for $F \sharp K$ with the same $\theta$-weight. Hence, we have $t(F \sharp K)=2 n$ by a similar argument to the previous proof. 
<smiles>[Y]C([Y])C=C</smiles>

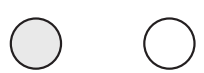

$\bigcirc$

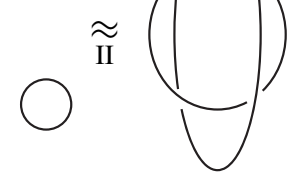

$t=-3$

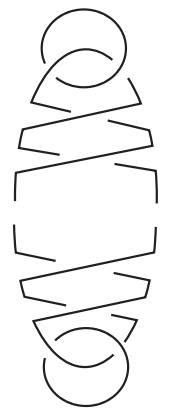

(i)

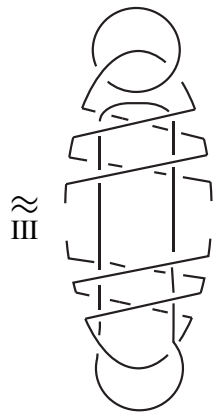

(ii)

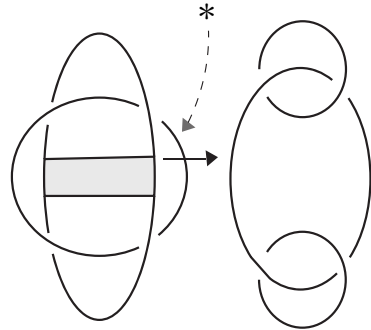

$t=-2$

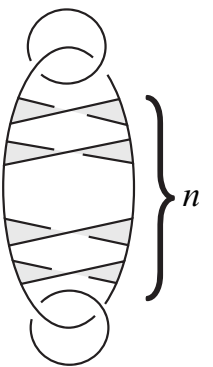

$t=-1$ $\approx$

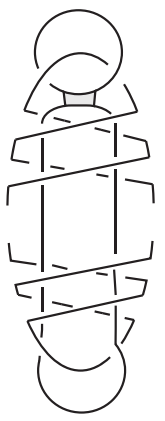

$t=0$

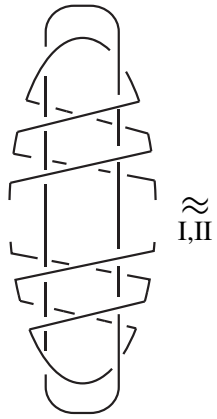

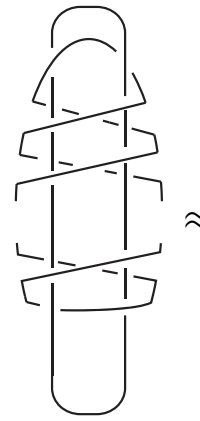

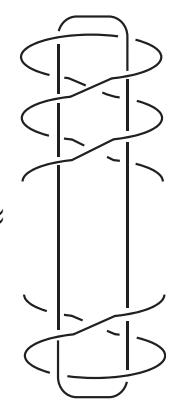

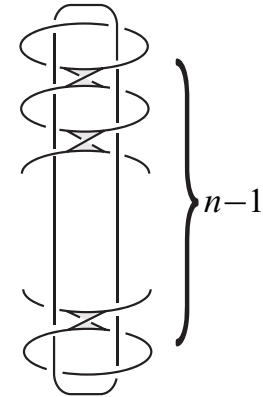

$t=1$

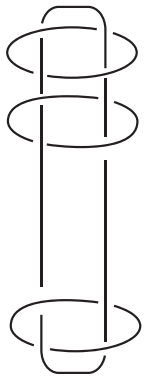

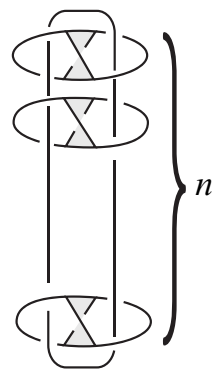

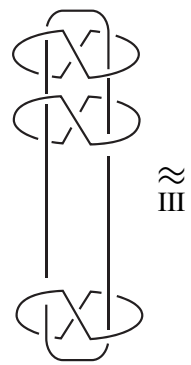

(iii)

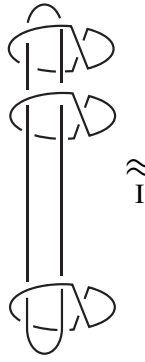

(iv)
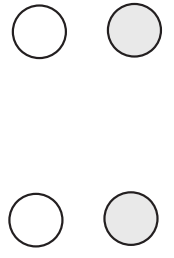

$t=3$

Figure 4

Remark 5.1 For the surface-link $F$ as above, we can also use Satoh's method [12] to prove that $t(F)=2 n$. However, for the surface-link $F \sharp K$ which is constructed in the proof of Theorem 1.3, we can not prove $t(F \sharp K)=2 n$ by his method since the surface-link is $P^{2}$-reducible. 

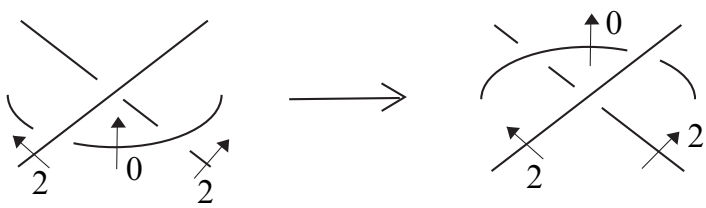

Figure 5
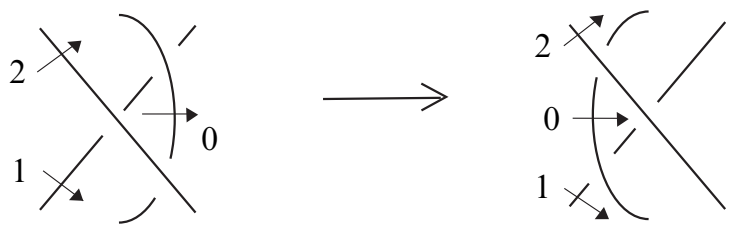

Figure 6

\section{Other results by Theorem 4.1}

In this section, we show some results which can be obtained as an application of Theorem 4.1.

For the positive integer $n$, let $G=G_{1} \cup G_{2}$ be a surface-link in $\mathbb{R}^{4}=\left\{(x, y, z, t) \in \mathbb{R}^{4}\right\}$ whose motion picture is given in Figure 7. Each component of $G_{i}$ is a non-orientable surface with $\chi\left(G_{i}\right)=2-n$. This is the surface-link which Satoh used for proving Theorem 1.1.

The following theorem is a generalization of Theorem 1.1. We can give alternative proofs by a symmetric quandle 3-cocycle similarly to the proof of Theorem 1.2, or by a geometric argument used in [12]. We say that a surface-link is pseudo-ribbon if it has a diagram without triple points (see Kawauchi [9]).
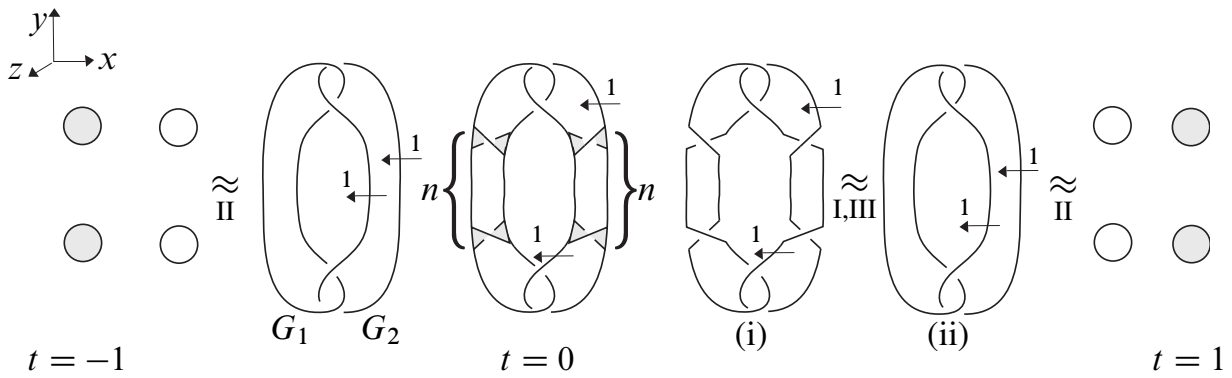

Figure 7 
Theorem 6.1 (Kamada and Oshiro [8]) Let $G$ be the surface-link as above. For any orientable surface-knot $K$, the connected sum $G \sharp K=\left(G_{1} \sharp K\right) \cup G_{2}$ satisfies

$$
t(G \sharp K) \geq 2 n \text {. }
$$

In particular, if $K$ is pseudo-ribbon, then the equality holds.

For a non-orientable surface-knot $K$, the connected sum $G \sharp K$ is not necessarily $P^{2}-$ irreducible. Hence we can not apply the Satoh's argument to the surface-link. In this case, we have the following.

Theorem 6.2 Let $G$ be the surface-link as above. For any non-orientable surface-knot $K$, it holds that

$$
t(G \sharp K) \geq\left\{\begin{array}{cl}
n+1 & \text { if } n \text { is an odd number, } \\
n & \text { if } n \text { is an even number. }
\end{array}\right.
$$

Proof Let $(X, \rho)$ and $\theta$ be the symmetric quandle and the symmetric quandle 3cocycle given in Example 2.2, respectively. By the definition,

$$
\theta(a, b, c) \in\{0 \oplus 0,1 \oplus 0,0 \oplus 1,0 \oplus(-1)\}
$$

for any $(a, b, c) \in X^{3}$.

Let $D$ be the diagram of $G$ corresponding to the motion picture and $C$ the $(X, \rho)-$ coloring for $G$ as shown in Figure 7. Between the stills (i) and (ii), the Reidemeister moves III arise $2 n$ times. More precisely, a pair of moves III is depicted in Figure 8. The sum of the $\theta$-weights is equal to

$$
-\theta(1,0,1)+\theta(0,2,0)=0 \oplus 1+1 \oplus 0=1 \oplus 1,
$$

and hence, we have $\theta(G, C)=n \oplus n$.

For any non-orientable surface-knot $K$, we extend the $(X, \rho)$-coloring $C$ for $G$ to that for $G \sharp K$ such that $K$ is colored trivially. Then it follows by definition that $\theta(G \sharp K, C)=\theta(G, C)=n \oplus n$, and we have the conclusion by Theorem 4.1.

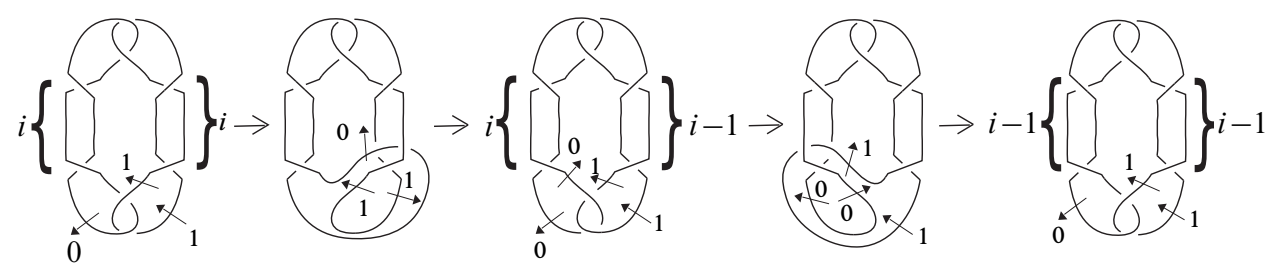

Figure 8 
The equality given in Theorem 6.2 holds for $n=1$.

Question 6.3 Does the equality in Theorem 6.2 hold for any $n \geq 2$ ?

We remark that the triple point number is generally not additive with respect to the connected sum (see Satoh [13]).

Acknowledgments The author is deeply grateful to Professor Seiichi Kamada for helpful suggestions and support. She would also like to thank Professor Shin Satoh for helpful comments about construction of surface-links. A part of this paper was written while she visited Professor Scott Carter and Professor Masahico Saito. She would also like to thank them for their helpful support and conduct. Finally, she would like to express her gratitude to the referee. This research is partially supported by Grant-in-Aid for JSPS Research Fellowships for Young Scientists.

\section{References}

[1] J S Carter, D Jelsovsky, S Kamada, L Langford, M Saito, Quandle cohomology and state-sum invariants of knotted curves and surfaces, Trans. Amer. Math. Soc. 355 (2003) 3947-3989 MR1990571

[2] J S Carter, M Saito, Knotted surfaces and their diagrams, Mathematical Surveys and Monographs 55, American Mathematical Society, Providence, RI (1998) MR1487374

[3] R Fenn, C Rourke, Racks and links in codimension two, J. Knot Theory Ramifications 1 (1992) 343-406 MR1194995

[4] E Hatakenaka, An estimate of the triple point numbers of surface-knots by quandle cocycle invariants, Topology Appl. 139 (2004) 129-144 MR2051101

[5] D Joyce, A classifying invariant of knots, the knot quandle, J. Pure Appl. Algebra 23 (1982) 37-65 MR638121

[6] S Kamada, Wirtinger presentations for higher-dimensional manifold knots obtained from diagrams, Fund. Math. 168 (2001) 105-112 MR1852735

[7] S Kamada, Quandles with good involutions, their homologies and knot invariants, from: "Intelligence of low dimensional topology 2006", Ser. Knots Everything 40, World Sci. Publ., Hackensack, NJ (2007) 101-108 MR2371714

[8] S Kamada, K Oshiro, Homology groups of symmetric quandles and cocycle invariants of links and surface-links, Trans. Amer. Math. Soc. (to appear)

[9] A Kawauchi, On pseudo-ribbon surface-links, J. Knot Theory Ramifications 11 (2002) 1043-1062 MR1941684 
[10] S V Matveev, Distributive groupoids in knot theory, Mat. Sb. (N.S.) 119(161) (1982) 78-88, 160 MR672410

[11] D Roseman, Reidemeister-type moves for surfaces in four-dimensional space, from: "Knot theory (Warsaw, 1995)", Banach Center Publ. 42, Polish Acad. Sci., Warsaw (1998) 347-380 MR1634466

[12] S Satoh, Minimal triple point numbers of some non-orientable surface-links, Pacific J. Math. 197 (2001) 213-221 MR1810216

[13] S Satoh, Non-additivity for triple point numbers on the connected sum of surface-knots, Proc. Amer. Math. Soc. 133 (2005) 613-616 MR2093086

[14] S Satoh, A Shima, The 2-twist-spun trefoil has the triple point number four, Trans. Amer. Math. Soc. 356 (2004) 1007-1024 MR1984465

Department of Mathematics, Hiroshima University

Higashi-Hiroshima, Hiroshima, 739-8526, Japan

d085317@hiroshima-u.ac.jp

Received: 22 April 2009 Revised: 21 November 2009 